\title{
Bio-imaging: late gadolinium enhancement in hypertrophic cardiomyopathy and its relation to novel biomarkers of fibrosis
}

\author{
Hassan Abdel-Aty ${ }^{2 *}$, Hugo A Katus ${ }^{1}$, Henning Steen', Stephanie Lehrke ${ }^{1}$ \\ From 15th Annual SCMR Scientific Sessions \\ Orlando, FL, USA. 2-5 February 2012
}

\section{Background}

Late gadolinium enhancement (LGE) accurately detects myocardial fibrosis in hypertrophic cardiomyopathy (HCM), which has been recently shown to be an independent risk factor for cardiac events in this setting. Endoglin is an accessory protein for the TGF- $\beta$ receptor, which stimulates myocardial fibrosis through modulating the response to Angiotensin II. We sought to explore the relationship between the myocardial fibrosis (tissue substrate) as identified by LGE and Endoglin (biomarker) in the setting of HCM.

\section{Methods}

We studied $133 \mathrm{HCM}$ patients (56 \pm 16 y, 94 men) using a whole body $1.5 \mathrm{~T}$ CMR scanner (Philips Achieva) with 32 channel image aquisition. Vector-ECG gated short axis, two and four chamber cine slices with parallel image aquisition covering the entire left ventricle (LV) were acquired using a regular SSFP sequence. Matched LGE images were acquired after IV Gadolinium DTPA $(0.2 \mathrm{mmol} / \mathrm{kg}$ bodyweight $)$ in all image planes. Two independent observers assessed LV volumes and myocardial mass that were acquired by manual drawing of endoand epicardial borders. LGE was defined as signal intensity increase of $>2$ SD of normal myocardium and after semi-automatic delineation on a commercially available workstation (Philips Viewforum) areas were quantified and expressed as percentage of the previously measured LV mass. Obstruction was determined using echocardiography gradient measurements at rest and under Valsalva.

$\mathrm{P}<0.05$ was considered statistically significant.

${ }^{2}$ Charité, Berlin, Germany

Full list of author information is available at the end of the article

\section{Results}

79 patients (59\%) exhibited intramural areas of LGE which averaged $7 \pm 12 \%$ of the LV mass. Patients with LGE had significantly higher levels of Endoglin compared with patients with no LGE lesions (3.8 \pm 0.4 vs. 2.4 $\pm 0.3 \mathrm{nl}, \mathrm{p}=0.018$ ). There was a weak but almost significant correlation between percent of LGE and Endoglin $(\mathrm{r}=0.16, \mathrm{p}=0.06)$. Endoglin levels however were not related to obstruction (HNCM: $3.7 \pm 0.4$ vs. $2.8 \pm 0.5$, $\mathrm{p}=\mathrm{ns})$ and were not related to age, gender or LV mass.

\section{Conclusions}

Our results provide preliminary evidence that Endoglin, a pro-fibrotic glycoprotein may be involved in the pathogenesis of late enhancement i.e. myocardial fibrosis in HCM. This molecular mechanism can have relevant clinical implications on the therapy of these patients since Endoglin expression (and thus probably myocardial fibrosis) can be suppressed using Angiotensin inhibitors although further studies on the prognosis and risk stratification of this new bio-imaging parameter have to be conducted.

\section{Funding}

None.

\section{Author details}

'Department of Cardiology, University of Heidelberg, Heidelberg, Germany.

${ }^{2}$ Charité, Berlin, Germany.

Published: 1 February 2012

doi:10.1186/1532-429X-14-S1-P164

Cite this article as: Abdel-Aty et al:: Bio-imaging: late gadolinium enhancement in hypertrophic cardiomyopathy and its relation to novel biomarkers of fibrosis. Journal of Cardiovascular Magnetic Resonance 2012 14(Suppl 1):P164. 